\title{
Correction to: Analysis of autonomic outcomes in APOLLO, a phase III trial of the RNAi therapeutic patisiran in patients with hereditary transthyretin-mediated amyloidosis
}

\author{
Alejandra González-Duarte ${ }^{1}$. John L. Berk ${ }^{2}$ - Dianna Quan ${ }^{3}$ - Michelle L. Mauermann ${ }^{4} \cdot$ Hartmut H. Schmidt ${ }^{5}$. \\ Michael Polydefkis ${ }^{6} \cdot$ Márcia Waddington-Cruz $^{7} \cdot$ Mitsuharu Ueda $^{8} \cdot$ Isabel M. Conceição ${ }^{9} \cdot$ Arnt V. Kristen $^{10}$. \\ Teresa Coelho ${ }^{11}$. Cécile A. Cauquil ${ }^{12}$. Céline Tard ${ }^{13} \cdot$ Madeline Merkel $^{14} \cdot$ Emre Aldinc $^{14}$. Jihong Chen ${ }^{14}$. \\ Marianne T. Sweetser ${ }^{14}$. Jing Jing Wang ${ }^{14}$. David Adams ${ }^{15}$
}

Published online: 7 February 2020

(c) The Author(s) 2020

\section{Correction to: Journal of Neurology} https://doi.org/10.1007/s00415-019-09602-8

The original version of this article unfortunately contained a mistake in Table 1.

Units for albumin were given as $\mathrm{g} / \mathrm{dL}$. It should be $\mathrm{g} / \mathrm{L}$.

Open Access This article is distributed under the terms of the Creative Commons Attribution 4.0 International License (http://creativeco mmons.org/licenses/by/4.0/), which permits unrestricted use, distribution, and reproduction in any medium, provided you give appropriate credit to the original author(s) and the source, provide a link to the Creative Commons license, and indicate if changes were made.

The original article can be found online at https://doi.org/10.1007/ s00415-019-09602-8.

Alejandra González-Duarte

gonzalezduarte@aol.com

1 Instituto Nacional de Ciencias Médicas Y Nutrición Salvador Zubirán, Vasco de Quiroga 15, Sección XVI, Tlalpan, CdMx, CP 01400 México City, Mexico

2 Boston Medical Center, Boston, MA, USA

3 University of Colorado, Denver, CO, USA

4 Mayo Clinic, Rochester, MN, USA

5 University of Münster, Münster, Germany

6 Johns Hopkins University School of Medicine, Baltimore, MD, USA

7 Hospital Universitário Clementino Fraga Filho-UFRJ, Rio de Janeiro, Brazil
8 Kumamoto University Hospital, Kumamoto, Japan

9 CHULN, Hospital de Santa Maria and Faculdade de Medicina, Universidade de Lisboa, Lisbon, Portugal

10 University of Heidelberg, Heidelberg, Germany

11 Hospital de Santo António, Centro Hospitalar Universitário Do Porto, Porto, Portugal

12 AP-HP Université Paris Saclay, CHU Bicêtre, Le Kremlin Bicêtre, France

13 Université de Lille, Lille, France

14 Alnylam Pharmaceuticals, Cambridge, MA, USA

15 AP-HP, Université Paris Saclay, CHU Bicêtre, Université Paris-Sud, INSERM 1195, Paris, France 
Table 1 Disease characteristics of autonomic endpoints at baseline

\begin{tabular}{|c|c|c|c|}
\hline Characteristic & Placebo $(n=77)$ & Patisiran $(n=148)$ & Total $(n=225)$ \\
\hline COMPASS-31 total score (range $0-100)$, mean $( \pm$ SD) & $30.3(16.4)$ & $30.6(17.6)$ & $30.5(17.1)$ \\
\hline Orthostatic intolerance (range: $0-40)$, mean $( \pm$ SD) & $13.2(11.2)$ & $14.2(10.8)$ & $13.8(10.9)$ \\
\hline Vasomotor (range $0-5)$, mean $( \pm \mathrm{SD})$ & $1.0(1.4)$ & $0.9(1.44)$ & $1.0(1.4)$ \\
\hline Secretomotor (range 0-15), mean $( \pm$ SD) & $4.9(3.7)$ & $4.2(3.7)$ & $4.4(3.7)$ \\
\hline Gastrointestinal (range 0-25), mean $( \pm$ SD) & $8.1(3.5)$ & $8.2(4.3)$ & $8.1(4.1)$ \\
\hline Bladder (range 0-10), mean ( \pm SD) & $1.9(2.7)$ & $2.0(2.5)$ & $2.0(2.5)$ \\
\hline Pupillomotor (range 0-5), mean ( \pm SD) & $1.1(1.1)$ & $1.2(1.2)$ & $1.2(1.2)$ \\
\hline $\mathrm{mBMI}\left(\mathrm{kg} / \mathrm{m}^{2} \times \mathrm{g} / \mathrm{L}\right)$, mean $( \pm \mathrm{SD})$ & $989.9(214.2)$ & $969.7(210.5)$ & $976.6(211.5)$ \\
\hline BMI $\left(\mathrm{kg} / \mathrm{m}^{2}\right)$, mean $( \pm \mathrm{SD})$ & $23.6(4.3)$ & $23.0(4.4)$ & $23.2(4.4)$ \\
\hline Albumin $(\mathrm{g} / \mathrm{L})$, mean $( \pm \mathrm{SD})$ & $41.8(3.4)$ & $42.1(3.5)$ & $42.0(3.5)$ \\
\hline Weight $(\mathrm{kg})$, mean $( \pm \mathrm{SD})$ & $67.5(15.7)$ & $67.3(16.6)$ & $67.4(16.3)$ \\
\hline mNIS +7 total score (range $0-304)$, mean $( \pm$ SD) & $74.6(37.0)$ & $80.9(41.5)$ & $78.8(40.1)$ \\
\hline Postural blood pressure (range $0-2$ ), mean ( \pm SD) & $0.6(0.7)$ & $0.7(0.8)$ & $0.6(0.8)$ \\
\hline Norfolk QOL-DN total score (range -4 to 136$)$, mean $( \pm$ SD) & $55.5(24.3)$ & $59.6(28.2)$ & $58.3(27.0)$ \\
\hline Autonomic neuropathy domain (range $0-12)$, mean $( \pm S D)$ & $2.9(2.9)$ & $3.0(2.8)$ & $3.0(2.8)$ \\
\hline
\end{tabular}

BMI body mass index, COMPASS-31 Composite Autonomic Symptom Score-31, $m B M I$ modified body mass index, $m N I S+7$ modified Neuropathy Impairment Score + 7, Norfolk $Q O L-D N$ Norfolk Quality of Life-Diabetic Neuropathy 